\title{
Pratiques
}

Linguistique, littérature, didactique

\section{Disciplinarité et instances de légitimation d'un domaine de recherche : la didactique du français et des langues au CNU}

\section{Dan Savatovsky}

\section{OpenEdition}

\section{Journals}

Édition électronique

URL : http://journals.openedition.org/pratiques/1686

DOI : $10.4000 /$ pratiques. 1686

ISSN : 2425-2042

Éditeur

Centre de recherche sur les médiations (CREM)

\section{Édition imprimée}

Date de publication : 15 juin 2011

Pagination : 25-40

Référence électronique

Dan Savatovsky, « Disciplinarité et instances de légitimation d'un domaine de recherche : la didactique du français et des langues au CNU », Pratiques [En ligne], 149-150 | 2011, mis en ligne le 16 juin 2014, consulté le 04 mai 2019. URL : http://journals.openedition.org/pratiques/1686 ; DOI : 10.4000/ pratiques. 1686 


\section{Disciplinarité et instances de légitimation d'un domaine de recherche : la didactique du français et des langues au CNU}

\section{Dan Savatovsky}

Université de Bourgogne - Univ. Paris Diderot, Sorbonne Paris Cité : laboratoire d'Histoire des Théories Linguistiques (UMR 7597 du CNRS)

Cet article vise à décrire sous certains aspects la disciplinarisation de la didactique du français et des langues (dorénavant: DdFL) en France, dans la période 2006-2011. Par disciplinarité, on entend d'abord - au sens étroit du terme - le poids, les formes ou les conditions de reconnaissance institutionnelle de la DdFL ; et par disciplinarisation les changements qu' elle a subis depuis une petite dizaine d'années - des changements qu'on mettra en perspective sur fond d'horizon plus lointain : le début des années 1980. Les données auxquelles on s'attache sont principalement les intitulés de thèses de doctorat figurant dans les dossiers de candidature soumis à la $7^{\mathrm{e}}$ section du Conseil national des universités (dorénavant : CNU) en vue d'une qualification sur la liste des maîtres de conférences (dorénavant: $\mathrm{MCF}$ ). Il s'agit d'indiquer certains des critères de reconnaissance et de délimitation de la didactique des langues, au premier chef celle du français, en vigueur au sein de l'une des principales instances de légitimation des travaux en sciences du langage (dorénavant : $\mathrm{SdL}$ ).

\section{État-civil disciplinaire}

La $7^{\mathrm{e}}$ section du CNU porte le nom de Sciences du langage : linguistique et phonétique générales. On n'évoquera pas ici les débats récurrents sur le sens ou la pertinence de cet intitulé. D'abord, sur le sens des deux désignations qui peuvent apparaître synonymes à certains membres de la communauté scientifique (mais pas à tous, précisément) : sciences du langage et linguistique. Sur le sens des deux points, ensuite, qui semblent exclure à l'inverse une glose en termes de doublons et présupposent que le genre $\mathrm{SdL}$ « coiffe » deux espèces distinctes : la linguistique, d'un côté, la phonétique de l'autre ; on se demande alors sur quels 
critères précis repose l'exigence de les distinguer et pourquoi il faudrait faire un sort particulier à la phonétique parmi tous les domaines qui relèvent des SdL. Sur la signification de "générale(s) », enfin : on voit bien qu'il s'agit là surtout de différencier la juridiction large de la section 7 de celles, plus étroites, des sections 11 (Langues et littératures anglaises et anglo-saxonnes), 12 (Langues et littératures germaniques et scandinaves), 13 (Langues et littératures slaves), 14 (Langues et littératures romanes) et 15 (Langues et littératures arabes, chinoises, japonaises, hébrä̈ques, d'autres domaines linguistiques). Mais, dans les faits, de nombreux candidats dont les travaux relèvent des sections CNU consacrées à des langues ou des familles de langues particulières (leurs membres se subdivisent traditionnellement en littéraires, linguistes et civilisationnistes) soumettent aussi - ou parfois seulement - leur dossier de qualification à la $7^{\mathrm{e}}$ quand leur thèse est à dominante linguistique. S'agissant à cet égard du domaine qui nous intéresse ici - la didactique d'autres langues que le français - les candidats qui envoient leur dossier à la $7^{\mathrm{e}}$ section représentent entre 4 et $8 \%$ de l'ensemble des dossiers, selon les années.

Quant à l'intitulé de la $7^{\mathrm{e}}$, on a assisté depuis qu'il a été fixé sous cette forme (au début des années 1980) à plusieurs types de démarches visant à le modifier - parfois à l'initiative de membres de la section; la proposition la plus fréquente étant de ne conserver que sciences du langage. Une autre proposition - émanant de didacticiens du FLE - et qui a fait l'objet d'une motion votée à une courte majorité par la section elle-même en 2007, revenait à lui substituer l'intitulé Sciences du langage et didactique des langues. Trois types d'arguments - peu compatibles entre eux - ont été avancés à l'encontre d'une telle proposition. (i) Comme à propos de la phonétique, pourquoi faire un sort à la didactique et ne pas énumérer alors l'ensemble des principaux domaines constitutifs des SdL (tâche qu'il serait imprudent d'entreprendre) ? (ii) La didactique ne ressortirait pas à la $7^{\mathrm{e}}$ section, mais à la $70^{\mathrm{e}}$ : ne pouvant l'exclure de la $7^{\mathrm{e}}$, on lui demande alors de se faire discrète en attendant que les didacticiens (ou du moins quelques uns d'entre eux, qui se recrutent surtout parmi les «FLEistes ») obtiennent ce qu'ils réclament - certains depuis longtemps : la création au CNU d'une section autonome de didactique des langues. (iii) Dernier argument, le plus décisif - nous semble-til - quand on s'attache vraiment aux intérêts de la didactique : sauf à le comprendre comme un hendyadis, le et qui conjoint didactique et $S d L$ fait peser un doute sur l'appartenance «naturelle» de la première aux secondes. Dissocier les deux domaines irait à l'encontre du but recherché : si le rattachement de la didactique des langues à la $7^{\mathrm{e}}$ section va sans dire, cela ne va pas mieux en le disant ${ }^{(1)}$.

Les enjeux auxquels renvoie le nom qu'il convient d'attribuer à la discipline impliquent donc plus largement les modalités d'inscription de la DdFL dans les SdL. L'examen des dossiers de candidature CNU peut sous cet aspect contribuer à préciser les frontières du domaine, qu'il s'agisse de ses frontières internes - comme les lignes de partage entre les champs du FLM, du FLE, du FLS et celui des autres langues ${ }^{(2)}$ - ou de ses frontières externes : les relations de voisinage

(1) Nul ne l'ignore : la motion votée est restée lettre morte.

(2) Respectivement: français langue maternelle, français langue étrangère et français langue seconde. Comme on sait, l'expression «français langue seconde » est ambiguë. Elle est parfois donnée pour synonyme de «français langue étrangère » quand elle désigne le français 
avec les domaines connexes - parmi lesquels ceux que la $7^{\mathrm{e}}$ section considère comme relevant «normalement» de sa juridiction (recherches en acquisition ou en cognition, psycholinguistique, sociolinguistique, etc.) ou comme n'en relevant pas (didactique de la littérature, sciences de l'éducation, etc.).

\section{Les autres facteurs de disciplinarité}

D'autres aspects de cette disciplinarité auraient mérité qu'on s'y attarde. Contentons-nous de les signaler brièvement. En vue d'ébaucher une typologie des objets de recherche en DdFL, il eût été ainsi utile de croiser les données issues des dossiers CNU avec d'autres données : celles qui ont trait à la politique éditoriale des revues de didactique, aux conditions d'accueil des travaux de DdFL par les revues généralistes en $\mathrm{SdL}$, par les revues spécialisées dans d'autres domaines des SdL ou dans d'autres domaines que les SdL (littérature et sciences de l'éducation, notamment), aux politiques de création d'emplois universitaires (profil " recherche » des fiches de poste), aux plans de formation universitaires (IUFM compris), aux orientations des écoles doctorales, etc.

Enfin, l'étude de la disciplinarisation de la DdFL eût gagné à prendre en compte d'autres facteurs - des facteurs qu'on ne peut, là aussi, qu'évoquer succinctement dans le cadre de cet article. Il s'agit en particulier des effets en retour que la politique linguistique ou éducative et la demande sociale exercent sur la structure et les orientations de la recherche. Il est probable sous cet aspect que le nombre relativement faible, parmi tous les dossiers soumis au CNU, des dossiers qui relèvent du FLM (entre 15 et $20 \%$ de l'ensemble DdF, selon les années) au regard de ceux qui relèvent du FLE s'explique par les modalités respectives de recrutement des enseignants (par concours, d'un côté, suivant le « libre » jeu du marché, de l'autre). Seuls $62 \%$ des candidats « FLEistes » à la qualification CNU en $7^{\mathrm{e}} \mathrm{sec}-$ tion (liste des MCF) pour la période qui nous intéresse, qu'ils aient soutenu leur thèse en France (la grande majorité) ou hors de France, sont détenteurs d'un master recherche (plus rarement professionnel) de ce type - mais en poursuivant un objectif le plus souvent directement professionnel. C'est en effet au niveau du master que recrutent désormais les institutions de diffusion du français à l'étranger (Alliances françaises, etc.). La plupart des autres candidats à la qualifica-

comme étant la première langue étrangère de l'élève ou de l'étudiant (donc la seconde après sa langue maternelle) dans un contexte où sa langue maternelle est dominante (c'est-à-dire, selon les cas, où elle est langue officielle, langue nationale ou langue majoritairement parlée dans un pays ou une aire linguistique donnés). Mais on a pris aussi l'habitude, depuis une quinzaine d'années, le plus souvent dans un contexte exolingue d'enseignement/apprentissage, de désigner plus spécifiquement par «FLS » le français tel qu'il est enseigné aux (ou pratiqué par les) locuteurs immigrés en France et dont la langue maternelle est autre que le français. Par extension, «FLS » réfère alors à un champ d'étude orienté vers (et un type d'enseignement destiné à) ces locuteurs-là, ainsi que - hors ou dans (1')institution scolaire - les structures éducatives qui leur sont adaptées (au sein des classes de CLIN dans l'enseignement primaire, ou des classes dites «d'accueil», dans les collèges, ou encore des cours d'alphabétisation destinés aux adultes, etc.). Le FLS ainsi compris a donné lieu à tout un ensemble de travaux portant spécialement sur la didactique et la pédagogie en vigueur dans ce type de classes ou de structures, en particulier des travaux consacrés au français comme «langue de scolarisation». 
tion, dont la thèse (en SdL, le plus souvent) porte sur la didactique du FLE ou du FLS, sont titulaires d'un master de type SdL généraliste - sans indication particulière de spécialité FLE - ou de type Littérature française ou Littérature comparée ( $22 \%$ pour ces trois catégories) ou bien d'un master de Langues, Littératures et Civilisations Étrangères (10\%). Ces indications sommaires sur le cursus d'origine des doctorants qui se sont spécialisés au niveau doctoral en didactique du FLE / FLS et visent une qualification CNU en $7^{\mathrm{e}}$ section reflètent assez mal - plus mal que dans d'autres domaines des $\mathrm{SdL}$ - la distribution par types de cursus antérieur de l'ensemble des docteurs ayant soutenu leur thèse - notamment en France - dans ce domaine. Un nombre important, difficile à évaluer (la majorité peut-être), de docteurs en SdL spécialisés en DdFLE ne se présentent pas à la qualification $\mathrm{CNU}$ en $7^{\mathrm{e}}$ section. Soit parce qu'ils soumettent leur dossier à une autre section $\mathrm{CNU}\left(9^{\mathrm{e}}, 10^{\mathrm{e}}, 70^{\mathrm{e}}\right)$, soit, pour la plupart, parce qu'il s'agit d'étudiants étrangers ou d'étudiants résidant à l'étranger qui ne visent pas une carrière universitaire en France.

Il serait utile de vérifier si, dans quelques années, la " mastérisation » du recrutement des professeurs de Lettres, ceux qui se destinent à l'enseignement secondaire, aura produit le même genre d'effets. On peut faire l'hypothèse d'une augmentation des flux de doctorants en didactique du FLM. Soit parce que des certifiés ou des agrégés de Lettres en plus grand nombre, désormais détenteurs d'un master, poursuivront en thèse « sur leur lancée », en visant l'enseignement supérieur (comme MCF ou comme PRAG/PRCE détachés). Soit, beaucoup plus sûrement, parce qu'un certain nombre de « reçus-collés »-les étudiants qui auront réussi au master, mais échoué aux concours - chercheront à s'inscrire en doctorat, faute de mieux (pourrait-on dire) et dans une sorte de fuite en avant, afin de valoriser encore davantage leur formation sur le " marché » d'un enseignement secondaire public devenu « libre » en grande partie, c'est-à-dire recrutant en plus grand nombre des professeurs vacataires sous-payés.

Enfin, notre étude aurait exigé qu' on la mette en contexte à la lumière d'un tableau d'ensemble de l'organisation de la recherche en France (organisation de colloques, création d'écoles de pensée, de laboratoires, de sociétés savantes, etc.) et d'un aperçu sur la place qui y est faite au champ de la didactique ${ }^{(3)}$. Ce sera l'objet d'un autre travail.

\section{La didactique du français entre linguistique et sciences du langage}

La question de savoir si la didactique des langues et, en son sein, celle du français, s'est ou non constituée en domaine de recherche « fondamentale », un domaine autonome et reconnu comme tel parmi les SdL, a suscité en France un débat assez nourri depuis une vingtaine d'années. Ce débat est l'une des conséquences lointaines de la reconfiguration institutionnelle intervenue au début des années 1980. Dans la période qui a suivi le premier rapport Godelier ${ }^{(4)}$ consacré $^{2}$

(3) On trouvera des indications précieuses sur toutes ces questions dans Chevalier et Encrevé (2006).

(4) Il serait utile de confronter la situation actuelle non seulement au constat dressé dans le rapport Godelier de 1982, mais également aux brèves indications consacrées aux SdL dans ce- 
aux Sciences de l'homme et de la société en France (1982) ${ }^{(5)}$, l'expression " sciences du langage » s'est assez rapidement substituée, on le sait, à celle de « linguistique », tout particulièrement là où se déroulaient les études - départements universitaires, écoles doctorales - et où se décidaient les carrières : sections du CNU, commissions de spécialistes, commissions du CNRS.

Un tel changement d'appellation, intervenu dans le cadre d'une reconfiguration d'ensemble du secteur des sciences humaines au sein des organismes de recherche, paraissait consacrer une conception scientiste de la discipline. Mais la nouveauté de l'expression (elle était nouvelle en France, du moins) ${ }^{(6)}$ tenait surtout au pluriel («les sciences du langage»), car on disait déjà «la science du langage », au milieu du XIX ${ }^{\mathrm{e}}$ siècle, avant que ne soit pleinement consacré le terme de linguistique. Cette dénomination répondait à la volonté d'ouvrir la discipline ainsi désignée à des champs d'étude diversement reconnus jusqu'alors, mais en plein développement (pragmatique, linguistique de l'énonciation, analyse du discours, neurosciences, etc.) et d'y accueillir des praticiens qui ne fussent pas seulement, voire pas du tout, des enseignants : professionnels de la santé hospitaliers ou libéraux (orthophonistes, phoniatres, psychothérapeutes, etc.) et travailleurs des industries de la langue (lexicographes, ergonomes du langage, spécialistes du dialogue homme/machine, etc.). Qu'importe ici si l'appel, lancé lors des Assises de la linguistique, à un élargissement des Sd L aux « métiers de la langue » n'a pas vraiment rencontré le succès escompté ${ }^{(7)}$. Dans l'ensemble, la greffe du pratico-technique, qui faisait partie des opérations programmées au début des années 1980, n'a pas pris. Ainsi, les secteurs de la santé ou des industries de la langue demeurent très minoritairement représentés parmi les docteurs candidats à une qualification $\mathrm{CNU}$ en $7^{\mathrm{e}}$ ( $2 \%$ pour la période de référence).

\section{Une situation instable}

Dans ce contexte, la situation de la didactique des langues apparaît assez instable. Le rapport Godelier qui reposait sur une enquête, entendait donner une

lui dont M. Godelier a dirigé la rédaction vingt ans plus tard, (Godelier et al., 2002). C'est à A. Peyraube qu'on doit le chapitre «Sciences du langage » de ce second rapport.

(5) C'est à B.-N. Grunig qu'était confié le chapitre consacré à la linguistique (pp. 421-446 du rapport). La dénomination «sciences du langage »n'a pas fait (et continue du reste de ne pas faire) consensus. Un signe parmi tant d'autres de la reconduction des clivages : dans le prolongement des Assises de la linguistique qui avaient notamment pour objet de débattre le plus largement possible de la politique de recherche dessinée dans le rapport Godelier, s'est créée en 1983 l'Association des Sciences du langage (ASL). Au même moment se créait une autre association professionnelle du même type, 1'Association des Linguistes de l'Enseignement Supérieur (ALES) qui, par son titre même, se différenciait doublement de l'ASL : il y était encore question de linguistes et donc de linguistique; et son recrutement était en principe restreint à l'enseignement supérieur - alors que celui de l'ASL se voulait plus large, incluant d'autres praticiens que les enseignants, d'autres enseignants que les universitaires et d'autres universitaires que les représentants du «noyau dur » (Godelier et al. : 431). Et parmi ces «autres», il faut certainement compter les didacticiens des langues.

(6) Comme on sait, depuis le début du XIX ${ }^{\mathrm{e}}$ siècle, les Allemands parlent plus volontiers de Sprachwissenschaft(en) que de Linguistik.

(7) De manière un peu imprudente, B.-N. Grunig «croy(ait) pouvoir dire que les linguistes n'aur(aient) aucune difficulté particulière pour répondre ce qu'il est convenu d'appeler la demande sociale » (ibid. : 433). 
image fidèle ${ }^{(8)}$ de la communauté des linguistes français du début des années 80 . Mais le statut disciplinaire de la didactique des langues n'y était pas clairement assigné à partir du moment où l'on refusait à la fois de la réduire à un type technico-pratique particulier (c'est-à-dire à un enseignement des langues non adossé à la recherche) et d'identifier son domaine d'appartenance comme étant celui de la linguistique appliquée ${ }^{(9)}$. Tout en associant les « recherches sur l'apprentissage et l'enseignement des langues maternelles et secondes » $\left(\right.$ op . cit., p. 427) ${ }^{(10)}$ à celles de la sociolinguistique avec laquelle elles auraient « une large intersection » (ibid.) et en dénonçant leur « situation institutionnelle proprement scandaleuse » (ibid. : 428), le rapport Godelier dressait un tableau très sombre de l'état de ces recherches, surtout en ce qui concerne le $\mathrm{FLM}^{(11)}$. La formation en didactique ayant cette particularité, parmi toutes les formations professionnelles menées à l'université, d'être fortement endogène ou plutôt auto-reproductrice (des enseignants y forment de futurs enseignants), intenter un procès en légitimité aux recherches menées dans son cadre entraîne presque mécaniquement qu'on dénie, pour l'essentiel, une légitimité à la formation elle-même ${ }^{(12)}$. C'est pour-

(8) Les questionnaires élaborés par M. Godelier et B.-N. Grunig, envoyés à 1000 chercheurs et enseignants de linguistique, avaient suscité plus de 600 réponses, le plus souvent sous forme d'envois groupés (ibid. : 423).

(9) On se félicite ainsi dans le rapport que M. Candelier ait « fait avancer la réflexion d'ensemble sur le statut même de la linguistique prétendue "appliquée” " (ibid. : 440, note 15).

(10) Les rédacteurs du rapport ne reprennent pas à leur compte le terme même de didactique. Par ailleurs, ils emploient les expressions «français comme langue étrangère » (ou « comme langue maternelle »), alors que français langue étrangère et français langue maternelle (1e plus souvent sous forme de sigles - FLM et FLE) sont déjà largement répandus dans les travaux des didacticiens. Il s'agit là d'un simple indice, mais il est significatif : le figement, l'opacité des formules FLM et FLE marquent aussi qu'une dénomination académique est arrêtée et que le champ d'enseignement et de recherche qu'elle recouvre est en passe de détenir la consistance d'une discipline (voir Berré et Savatovsky, éds, $2010: 5$ ).

(11) «Peut-être les recherches des psychologues ou celles des sciences de l'éducation recèlentelles, en ce qui concerne l'apprentissage et l'enseignement du français langue maternelle, des ressources que nous ne soupçonnons pas. Sans doute n'avons-nous pas eu la possibilité d'atteindre véritablement les cercles des CRDP, CDDP et EPN. Moyennant ces réserves, nous devons dire ici que l'impression d'ensemble que nous livre notre consultation est désolante en ce qui concerne la recherche institutionnelle dans ce domaine » (op. cit. : 439, note 15). Le paysage des recherches portant sur le FLE apparaît cependant un peu moins désolé à travers le rapport, mais avec une curieuse assignation des motifs de cette spécificité sur le terrain de la «théorie »: «dans ce second domaine, les équipes ont, semble-t-il, pu mieux survivre au sein de la débâcle institutionnelle. Cela tient peut-être pour une part au fait que [...] les problèmes théoriques sont un peu moins redoutables (on part déjà d'une autre langue, "sue") » (ibid.). Et de citer, parmi les organismes de recherche reconnus en DdFle, le CREDIF (D. Coste, J. Cortès, H. Besse, L. Porcher), le BELC (F. Debyser), le Centre de linguistique appliquée de Besançon (E. Lhote), le Centre de didactique des langues de Grenoble 3 (L. Dabène), le GRAL de Paris 8 (C. Noyau, J. Arditty) ou « le groupe d'Aix » (D. Véronique, D. Faita). De manière générale, le tableau de la recherche en didactique, peint comme un champ de ruines, tel qu'on le trouve dans le rapport Godelier, ne va pas sans un certain paradoxe : le moins qu'on puisse dire, est que la liste des équipes et des groupes qui s'y consacrent directement ou indirectement apparaît - parmi tous les domaines évoqués - comme étant la plus longue.

(12) Voir faire et faire voir sont, en fin de compte, les deux seules modalités de formation des enseignants auxquelles les adversaires de la didactique (de manière générale) accordent quelque crédit. Tout en ramenant « ce qu'il est convenu d'appeler la socio-linguistique » (ibid. : 427) - domaine auquel serait lié de manière privilégiée l'enseignement/ apprentissage des langues - à l'une des « quatre forces » à l'œuvre en linguistique en ce début des années 80 , la «force communicationnelle », le rapport Godelier borne très précisément l'action du lin- 
quoi les didacticiens du français qui se sont efforcés, durant la même période, de constituer leur spécialité en champ disciplinaire autonome ${ }^{(13)}$, avec des modalités distinctes selon qu' on avait affaire au FLM ou au FLE, ont cherché à déconnecter la recherche des préoccupations professionnelles immédiates. Ils l'ont cherché le plus souvent en refusant précisément l'idée qu'il faille continuer à identifier la didactique des langues comme un secteur de la linguistique appliquée ${ }^{(14)}$.

La mutation des années 1980, les écarts d'interprétation du degré de reconnaissance accordé à la DdFL selon qu'elle est vue de l'extérieur ou de l'intérieur du domaine, requièrent donc qu' on y porte un regard rétrospectif en s'attachant à retracer le trajet qui a précisément mené de la «linguistique » aux «sciences du langage » et permis à la didactique d'acquérir in fine une certaine assise institutionnelle, celle dont témoigne l'actuelle «politique » du CNU à son égard. Mais la place reconnue à la DdFL dans ce trajet ne peut s'apprécier seule. Elle ne peut l'être qu'à l'aune des rapports qu'institue entre tous les domaines des SdL cette politique de légitimation menée au CNU. Les taxinomies sur lesquelles elle repose implicitement dessinent une cartographie en acte de la discipline. Se pose alors notamment la question de savoir ce qui, dans les relations qu'ils entretiennent, permet de distinguer, à la lumière de leur histoire récente, les domaines que la nouvelle configuration a pris en charge de ceux qui n'y ont pas (ou pas complètement) trouvé leur place ? C'est là sans doute aussi que s'est joué le sort de la didactique des langues dans la période récente. L'autonomie de la DdFL est donc également une affaire de frontières.

\section{Frontières}

La question des frontières est une question stratégique pour une discipline et qui occupe une place centrale dans son horizon de prospection. C'est aussi une question décisive pour l'historiographe des disciplines, tout particulièrement lorsqu'il s'attache aux objets frontaliers que je viens d'évoquer. L'histoire de la linguistique et de la didactique - fût-ce celle du temps présent-nous montre que

guiste dans la sphère scolaire : il «pourra apporter une contribution à la rénovation de l'école, à la compréhension des échanges verbaux quotidiens [...], mais qu'on ne lui demande pas de recettes pour une "bonne" communication, elles ne seraient qu'illusoires. " (ibid. : 433).

(13) Mouvement enclenché antérieurement, bien entendu, mais qui se réinscrit alors dans la reconfiguration générale du domaine. Dans le champ de la DdF, une revue comme Études de linguistique appliquée, revue internationale d'applications linguistiques et de didactique des langues, créée en 1961, a toujours été dirigée par des linguistes (son fondateur, B. Quemada, est lexicologue / lexicographe) surtout préoccupés, comme l'indique le titre de la revue, par les applications à l'enseignement. Cette orientation presque exclusivement didactique (qui la différencie, par exemple, de la Revue française de linguistique appliquée, fondée plus tard, en 1996) se précise en 1991, avec un fort tropisme FLE, quand, à l'initiative de son directeur, R. Galisson, la revue change de titre pour devenir Revue de didactologie et de lexiculturologie des langues-cultures, tout en gardant son premier titre en sous-titre. Deux revues qui font paraître majoritairement des travaux de didactique du FLM, comme Le Français aujourd'hui, et Pratiques, cette dernière publiée par un «collectif de recherche et d'expérimentation sur l'enseignement du français » animé par A. Petitjean, avaient été fondées respectivement en 1969 et 1974.

(14) S'agissant de l'histoire récente des rapports entre didactique et linguistique appliquée et de leurs avatars, voir Coste (2011). 
la question des frontières externes apparaît plus sensible lorsqu'à la suite d'une crise intestine $^{(15)}$, s'est manifestée l'exigence d'intégrer de nouveaux objets de savoir, donc de redessiner les lignes de démarcation internes à la discipline. Les débats sur le périmètre consenti à la didactique du français et des langues en son sein sont donc, à cet égard aussi, un assez bon indice de l'état général des SdL, des mouvements qui tendent à inclure/exclure les domaines jugés frontaliers et/ou marginaux. Dans ce contexte, les orientations de recherche des docteurs candidats à la qualification forment, à trente ans d'écart, on ne s'en étonnera pas, un indicateur fiable de ces mouvements, avec des effets de retard par rapport à leur déclenchement supposé. Sans doute, chaque membre de la $7^{\mathrm{e}}$ section n'a-t-il à expertiser qu'une vingtaine de candidatures MCF parmi les quelque 240 à 300 effectivement examinées tous les ans - et même moins, s'agissant des membres appartenant au corps A (professeurs des universités) ${ }^{(16)}$. Mais la lecture des rapports en session permet à l'ensemble de la section de se faire une idée raisonnable du contenu de chaque dossier.

Dans ce type de dossier, la thèse de doctorat est l'indicateur le plus fiable du domaine de spécialité des candidats ${ }^{(17)}$. On peut se fonder sur le titre de ces thèses pour opérer un classement des recherches doctorales par sous-domaines disciplinaires ${ }^{(18)}$. Le dépouillement du fichier central des thèses soutenues (ou en cours de réalisation) dans les universités françaises aurait certainement abouti à un résultat plus fidèle - chaque intitulé y est assorti d'un résumé - et surtout plus complet : tous les doctorants ne deviennent pas docteurs et tous les docteurs ne soumettent pas de dossier au CNU. Mais les titres de thèses mentionnés dans les dossiers CNU, présentent un intérêt différent. D'une part, les candidats à la qualification n'ont pas tous, loin de là, réalisé leur doctorat dans une université française ; d'autre part, un nombre non négligeable d'entre eux ne viennent pas des $\mathrm{SdL}, \mathrm{n}$ 'ont pas mené leur recherche dans une école doctorale de linguistique ou sous la direction d'un linguiste ${ }^{(19)}$. La classification par domaines ici proposée, si elle ne donne qu'une image incomplète de l'état des recherches doctorales françaises en SdL, reflète donc surtout l'étendue de la juridiction de cette disci-

(15) Le chapitre « Linguistique » du rapport Godelier partait du constat « d'une crise (de la discipline) dont il (fallait) savoir saisir au vol le potentiel dynamique » (op. cit. : 425).

(16) Candidatures en vue de la liste MCF et de la liste professeurs confondues. Les membres du collège A examinent à la fois les dossiers professeurs et MCF; les membres du collège B, pour leur part, n'examinent que les dossiers MCF. La $7^{\mathrm{e}}$ section comprend 36 membres et chaque dossier est confié à deux rapporteurs. Si les deux rapporteurs s'accordent, soit à préconiser la qualification, soit à proposer de la refuser, la section entérine dans la presque totalité des cas. Si les deux rapports divergent, la section vote. Mais tout membre de la section peut demander un vote, même lorsque les rapporteurs ont émis des avis convergents, et il a pu arriver qu'à l'issue d'une discussion générale ceux-ci ne soient pas suivis.

(17) Il est moins fiable, on l'imagine, pour ce qui est de la qualification aux fonctions de professeur, dans la mesure où de nombreux candidats, le plus souvent déjà MCF quand ils soumettent leur dossier, ont une partie de leur carrière et de leur production scientifique derrière eux et où certains d'entre eux ont pu réorienter leurs recherches dans des directions, dans des domaines plus ou moins éloignés de celui de leur thèse. Mais cela est vrai aussi en partie pour les candidats à la qualification MCF dont la thèse est déjà ancienne et qui demandent un renouvellement de qualification.

(18) J'ai proposé ailleurs un tel classement, mais limité à la campagne de qualification 2006 (Savatovsky, 2007). Je reprends ici, en les développant, certaines des analyses alors publiées.

(19) La section requiert qu'au moins l'un des membres du jury (et pas nécessairement le directeur) de thèse relève de la $7^{\mathrm{e}}$ section pour que la qualification soit envisageable. 
pline en France, telle que les candidats se la représentent : en adressant leur dossier à la $7^{\mathrm{e}}$ section, en effet, ils le présument qualifiable en SdL. Quant à la répartition qualifiés / non qualifiés qui figure aussi dans le tableau ci-dessous (pages

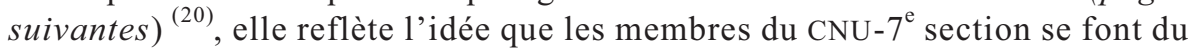
degré d'appartenance légitime aux SdL de telle ou telle spécialité universitaire ; or c'est là leur principal critère de sélection.

\section{Classification, interfaces et regroupements}

Cette classification a ses limites. Pour aller plus loin, il faudrait s'attacher aux années antérieures, de façon à pouvoir relever d'éventuelles évolutions ${ }^{(21)}$. Il faudrait surtout réfléchir plus rigoureusement aux critères de catégorisation par domaines de spécialité eux-mêmes. Ceux dont on s'est servi présentent plusieurs défauts évidents. Il y a des regroupements discutables : ainsi, pourquoi associer les travaux qui relèvent de la neurolinguistique et ceux qui relèvent de la psycholinguistique, qui portent sur l'acquisition ou les pathologies du langage? Pourquoi dissocier acquisition et didactique ? Autre exemple : pourquoi dissocier stylistique et analyse du discours qui convergent dans de nombreux cas - mais pas dans tous les cas, loin de là - si du moins l'on admet que la stylistique n'a pas trait seulement aux textes littéraires (mais aussi au langage ordinaire), que l'analyse du discours n'a pas trait seulement au discours oral et que l'un et l'autre de ces domaines peuvent, dans certains cas, porter conjointement sur les procédés d'argumentation, les diverses formes de la présupposition, etc. (mais alors pourquoi les dissocier à leur tour de la sémantique et/ou de la pragmatique et/ou du domaine de l'énonciation ?). Difficultés sans fin... Il est ainsi possible qu'en matière de DdF il vaille mieux ne pas regrouper FLM et FLE. Ce serait pourtant sans compter que certains travaux sont à cheval sur les deux domaines. Mais surtout, même lorsqu'ils sont eux-mêmes spécialistes du FLE ou du FLM, comme c'est le cas depuis 2009 pour deux d'entre eux, les membres du bureau de la $7^{\mathrm{e}}$ section considèrent que les différences FLE / FLM ne sont pas suffisamment marquées au regard de la principale mission du CNU (la qualification) pour attribuer les dossiers relevant de chacun des deux champs à des catégories distinctes de rapporteurs ${ }^{(22)}$.

(20) Faute de place, nous ne présentons que les données détaillées des années 2006 (l'avant-dernière de l'exercice 2004-2007), 2008 et 2011 (la première et la dernière de l'exercice 20082011). Ces trois jalons permettent néanmoins de se faire, à la fois, une idée des changements intervenus dans la politique de qualification de la $7^{\mathrm{e}}$ section et des évolutions récentes en matière de recherches doctorales (par domaine, par langue ou familles de langues) s'agissant bien sûr des thèses entreprises entre trois et six/sept ans auparavant. $88 \%$ des thèses figurant dans les dossiers de qualification ont été soutenues l'année même ou bien l'une des deux années précédant l'envoi du dossier au CNU ; les thèses plus anciennement soutenues correspondent pour l'essentiel à des demandes de nouvelle qualification.

(21) M. Arrivé (2000), alors président de la $7^{\mathrm{e}}$ section, avait rendu compte des principaux résultats de la première campagne de qualification de l'exercice 2000-2003. Les tendances qu'il dégageait se sont trouvées globalement confirmées au cours des deux exercices suivants, s'agissant en particulier de l'augmentation du nombre des recherches en didactique des langues.

(22) Douze des trente-six membres de la $7^{\mathrm{e}}$ section déclarent la didactique du français et/ou des langues comme étant ou comme faisant partie de leur(s) domaine(s) de recherche. 


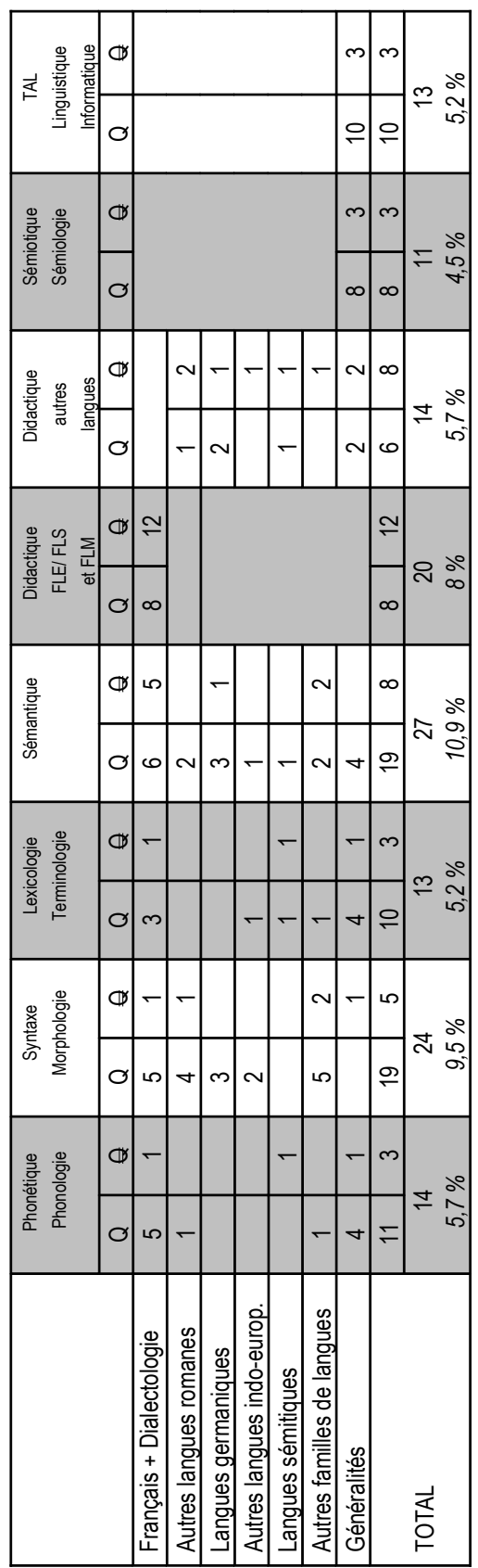

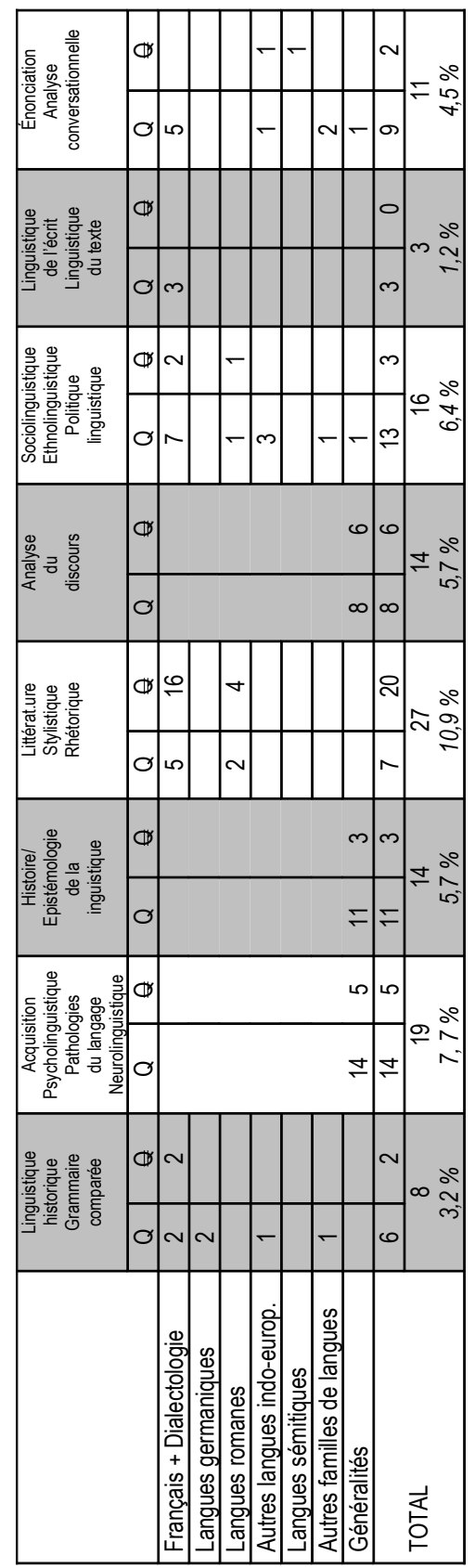

\section{$\mathrm{CNU} / 7^{\mathrm{e}}$ section - Campagne de qualification 2006 (MCF)}

Répartition des dossiers de candidature par domaines de spécialité (d'après l'intitulé de la thèse) Total des candidats inscrits via ANTARES $=329$

Dossiers examinés $=248=66,4 \%$. [Dossiers non parvenus, parvenus hors délais ou irrecevables $=77=23,6 \%]$

Candidats qualifiés par la $7^{\mathrm{e}}$ section $(\mathrm{Q})=161=65,5 \%$ des dossiers examinés

Candidats non qualifiés par la $7^{\mathrm{e}}$ section $(\mathrm{Q})=87=34,5 \%$ des dossiers examinés 

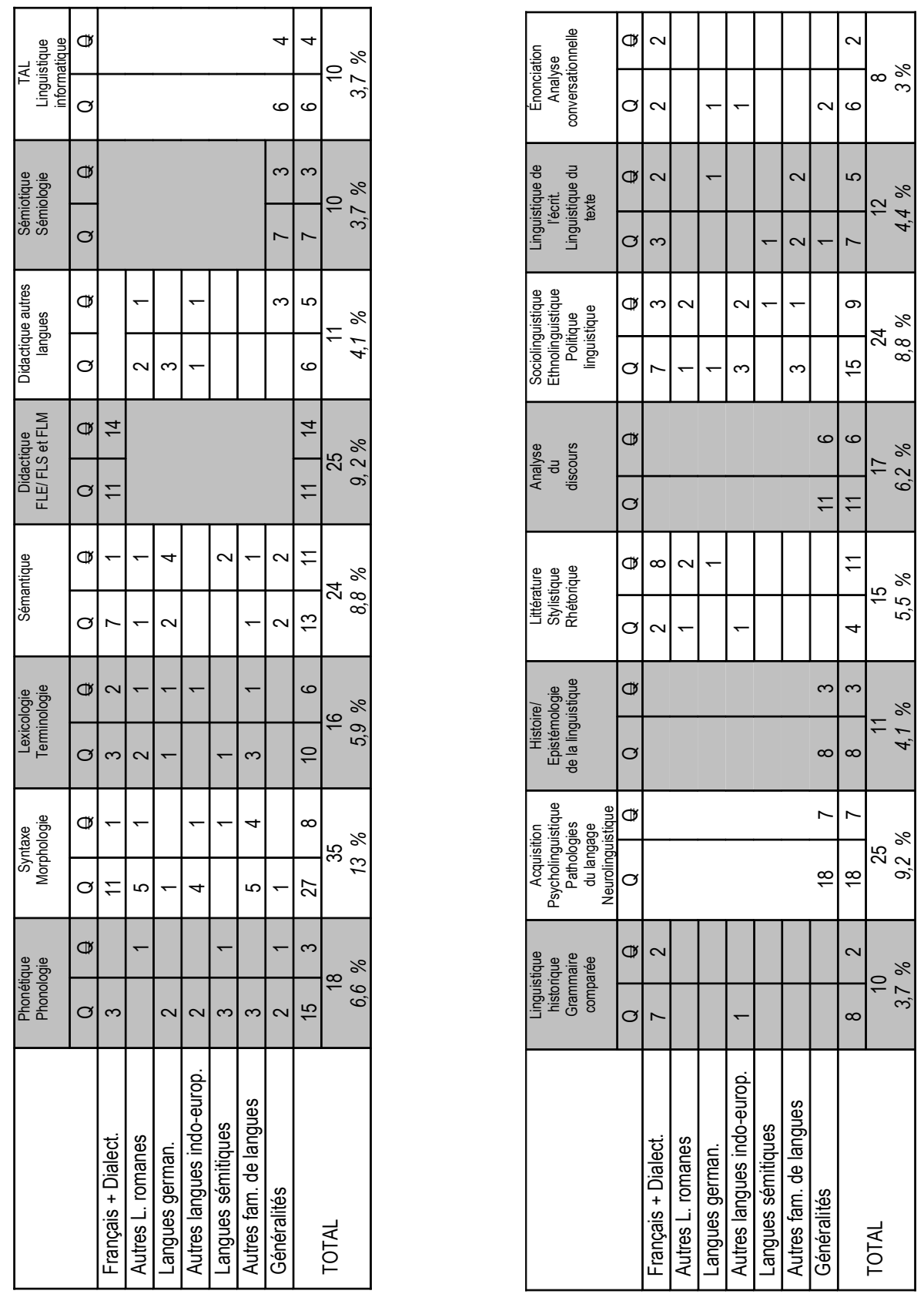

\section{CNU/7 $7^{\text {e }}$ section - Campagne de qualification 2008 (MCF)}

Classement des dossiers de candidature par domaines de spécialité (d'après l'intitulé de la thèse). Total des candidats inscrits via ANTARES $=331$

Dossiers examinés $=270=81,5 \%$. [Dossiers non parvenus, parvenus hors délais ou irrecevables $=61=18,4 \%]$

Candidats qualifiés par la $7^{\mathrm{e}}$ section $(\mathrm{Q})=165=61,1 \%$ des dossiers examinés

Candidats non qualifiés par la $7^{\mathrm{e}}$ section $(Q)=105=38,9 \%$ des dossiers examinés 

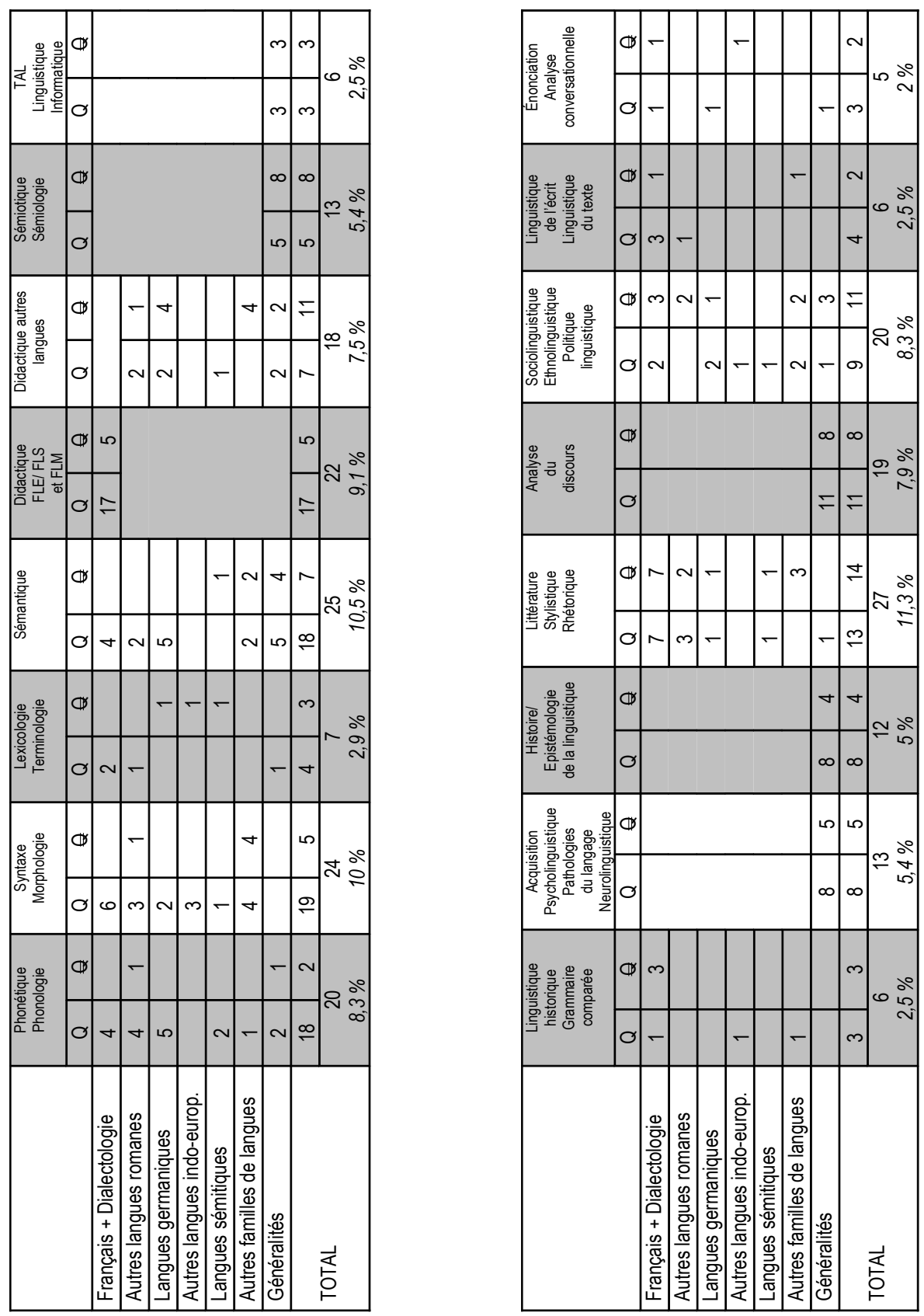

$\mathrm{CNU} / 7^{\mathrm{e}}$ section - Campagne de qualification 2011 (MCF)

Répartition des dossiers de candidature par domaines de spécialité (d'après l'intitulé de la thèse) Total des candidats inscrits via ANTARES $=285$

Dossiers examinés $=240=84,3 \%$. [Dossiers non parvenus, parvenus hors délais ou irrecevables $=45=15,7 \%]$

Candidats qualifiés par la $7^{\mathrm{e}}$ section $(\mathrm{Q})=149=62 \%$ des dossiers examinés

Candidats non qualifiés par la $7^{\mathrm{e}}$ section $(Q)=91=38 \%$ des dossiers examinés 
Autre défaut patent, s'agissant de la répartition qualifiés / non qualifiés, lié au fait même de n'avoir retenu comme données que les intitulés de thèse : 1'examen d'un dossier de candidature porte sur de nombreux aspects (rapport de thèse, composition du jury de soutenance, expérience d'enseignement, implication dans des équipes de recherche, enrichissement depuis quatre ans du volet «publications » quand il s'agit d'une demande de requalification, nature et support des publications, etc.). Le simple titre d'une thèse ne permet donc pas toujours de catégoriser le dossier de candidature ni même parfois le contenu de la thèse elle-même. Il ne le permet pas, soit parce que ce travail relève de plusieurs domaines à la fois (il a fallu alors choisir), soit parce que le titre est allusif - c'est parfois le cas quand il s'agit, selon toute apparence, de didactique, mais sans que la (ou les) langue(s) d'apprentissage soi(en)t clairement désignée(s) dans l'intitulé.

Dernier inconvénient principal : en cherchant à inclure dans le tableau la distribution des dossiers en qualifiés / non qualifiés, nous n'avons pas tenu compte des dossiers qui, pour une raison ou pour une autre, ne sont finalement pas parvenus aux rapporteurs désignés ou n'étaient pas recevables. Leur proportion n'est pas négligeable : entre $15 \%$ et $25 \%$, bon an mal an. Or, l'intitulé de la thèse de tous les candidats annoncés figure dans l'application ANTARES. Il serait alors sans doute utile de tenter de répartir l'ensemble des thèses par domaines de spécialité et pas seulement celles des candidats dont la section a eu effectivement à connaître. Un rapide aperçu sur ANTARES (en 2010 et 2011) montre que les domaines frontaliers, comme la didactique des langues, mais ni plus ni moins que la stylistique ou la linguistique informatique, sont ceux où des candidats ont eu la tentation de se présenter en $7^{\mathrm{e}}$ section, avant de se raviser et de ne pas envoyer leur dossier, plus souvent que lorsqu'il s'agit des autres domaines. Il n'y a pas de quoi en être surpris : les hésitations des membres de la section quant à l'appartenance au champ sont, dans l'ensemble, partagées par une partie des candidats eux-mêmes.

\section{Les refus de qualification}

Seconde remarque d'ordre général : le ratio candidats qualifiés / non qualifiés diffère de manière assez contrastée selon les domaines. On ne s'étonnera pas que certains de ceux à propos desquels « la section a été amenée à préciser sa spécificité » (Compte rendu de la $7^{e}$ section du CNU, qualifications 2005) ${ }^{(23)}$, comme la DdFL dans toutes ses composantes ou bien la stylistique française ${ }^{(24)}$, soient précisément les domaines où les refus de qualification sont à proportion les plus nombreux ${ }^{(25)}$. En tant que domaines frontaliers, ces deux domaines abritent des

(23) Compte rendu voté à l'unanimité des membres de la section, puis diffusé largement: presse syndicale, presse professionnelle (Buscila), page Internet de la CP-CNU sur le site du MEN, etc.

(24) «Littérature et stylistique françaises : ces champs relèvent normalement de la $9^{\mathrm{e}}$ section (de même pour les travaux littéraires sur d'autres langues, qui relèvent de la section concernée par cette langue). Pour qu'une qualification en $7^{\mathrm{e}}$ puisse être envisagée, une dimension d'analyse linguistique des textes doit apparaître clairement, notamment à travers la problématique, les références bibliographiques et le jury de thèse » (ibid.)

(25) En didactique (DdF et didactique d'autres langues confondues), $50 \%$ en moyenne des dossiers ne sont pas qualifiés sur l'ensemble de la période de référence. Mais cette proportion recouvre d'assez nettes disparités. Le nombre de dossiers non qualifiés passe de $58 \%$ en 2006 à $52 \%$ en 2008 et à $40 \%$ en 2011 . La tendance est donc plutôt favorable à la DdFL. 
travaux qui peuvent relever en partie ou totalement d'une autre discipline, donc d'une autre section du CNU : la $9^{\mathrm{e}}$ section (Littérature française), s'agissant de stylistique française, et la $70^{\mathrm{e}}$ (Sciences de l'éducation) ou les sections 11 à 15 , s'agissant de didactique des langues. Lorsqu'ils en relevaient totalement, et quelle que soit la qualité des dossiers, la $7^{\mathrm{e}}$ section a considéré qu'ils étaient hors champ et elle a refusé la qualification. Mais s'ils n'en relevaient qu'en partie et que « leur cadre théorique convoqu(ait) au moins un autre domaine des sciences du langage »(ibid.), la qualification a été accordée, toutes choses égales d'ailleurs.

On ne s'étonnera pas davantage qu' a contrario les dossiers relevant de la linguistique formelle, domaines anciennement disciplinarisés au sein des SdL et parfois identifiés comme faisant partie de leur «noyau dur»-c'est surtout le cas de la phonétique / phonologie, de la syntaxe et de la morphologie prises ensemble c'est-à-dire ceux dont l'identité disciplinaire ne fait guère de doute, soient aussi les dossiers qui proportionnellement ont fait le plus souvent l'objet d'une qualification $^{(26)}$. C'est également le cas, pour les mêmes raisons mais dans des proportions moindres, de la lexicologie et de la sémantique - un domaine devenu depuis quelques années celui dans lequel, devant la stylistique/rhétorique/recherche en littérature $(9,2 \%$ du total, sur la période étudiée) et la didactique du français et des langues $(7,2 \%$ du total, toutes langues confondues $)$ - le nombre de thèses figurant dans les dossiers présentés à la $7^{\mathrm{e}}$ section est le plus élevé (10\% du total).

Il est deux autres domaines pour lesquels le ratio dossiers qualifiés / non qualifiés est comparable à celui de la linguistique formelle : le TAL et l'histoire-épistémologie des sciences du langage. L'histoire-épistémologie ne fait plus partie des champs pour lesquels la section éprouve encore le besoin d'énoncer des conditions d'appartenance ${ }^{(27)}$. Le TAL exige que l'on distingue « une approche plus informatique et une autre plus linguistique. Le jury et les références bibliographiques ne doivent pas être exclusivement informatiques » (ibid.). De même, pour ce qui est de la sémiologie, où le taux de qualification est plus contrasté selon les années, elle a estimé nécessaire de préciser que «la recherche doit attester une maîtrise des concepts et méthodologies intéressant les sciences du langage et l'analyse des discours » (ibid.). Mais dans tous les cas, sauf en stylistique ${ }^{(28)}$,

(26) Linguistique historique ou grammaire comparée mises à part. Les dossiers comprenant une thèse et/ou des travaux en grammaire comparée (des langues indo-européennes) étant rarissimes, ils ont été regroupés avec ceux qui relevaient de la linguistique historique.

(27) Mis à part les domaines cités précédemment, font partie des champs dont les conditions d'appartenance aux sciences du langage sont soumises à restriction ou à précision dans le Compte rendu de la $7^{e}$ section : "langues étrangères et traduction 》 (les " thèses relevant d'une des sections de langues, il convient évidemment qu'elles traitent soit de problèmes linguistiques, soit de didactique. Pour la traduction, il doit s'agir de traductologie »); « sciences de l'éducation : [...] une recherche (qui en relève) ne peut être qualifiée en $7^{\mathrm{e}}$ section que si elle porte sur la didactique des langues (ou du français) et répond aux critères énoncés ci-dessus à propos de ce domaine»; enfin, les «sciences cognitives [qui] sont par nature interdisciplinaires. Il convient que la part accordée aux sciences du langage soit suffisamment importante ».

(28) C'est dans la catégorie «stylistique » que le taux de qualification est le plus faible, avec une nette tendance à s'affaiblir davantage encore dans les dernières années. La stylistique en tant que telle relève bien de la $7^{\mathrm{e}}$ section, mais de nombreux dossiers que nous avons rangés sous cette catégorie ressortissent en réalité à la $9^{\mathrm{e}}$ section (Littérature française) ou des sections 11 à 15 (composante littéraire). 
les candidats semblent désormais avoir une assez bonne connaissance des conditions auxquelles ils doivent souscrire pour que leurs travaux soient reconnus comme relevant de la discipline : ceux qui n'y souscrivent pas sont moins nombreux que naguère à soumettre leur dossier à la $7^{\mathrm{e}}$ section. Il en va de même, si l'on considère les choses en tendance, des dossiers de didactique : après une période (2003-2007), où le nombre de refus de qualification est demeuré relativement stable, à un niveau assez élevé, il a tendance à baisser.

Cela n'est pas entièrement vrai de l'analyse de discours, domaine dans lequel la proportion qualifiés / non qualifiés est pour ainsi dire en équilibre et n'a guère varié depuis six ans. Mais il est vrai que la délimitation des frontières de l'analyse du discours avec les domaines connexes (selon les types de travaux) de la sociolinguistique ou de la stylistique/rhétorique, voire de la sémantique, pose problème (elle pose d'abord problème aux chercheurs en analyse du discours); la présentation de ce champ dans le tableau a pu s'en trouver biaisée. On peut faire une remarque analogue s'agissant de tous les travaux situés à l'interface de deux domaines de spécialité : syntaxe / sémantique ou sémantique / lexicologie ou bien énonciation / rhétorique.

Toutes ces difficultés ne sont, à l'évidence, pas étrangères au sort qui est fait à la didactique des langues. Le recours à une approche interdisciplinaire, la figuration des interfaces sont des facteurs qui entraînent plus souvent une qualification quand les thèses en didactique des langues font appel aux méthodes et aux descriptions de l'analyse formelle (le « noyau dur ») ou sémantique qu'à celles de l'analyse du discours, de l'analyse conversationnelle ou de la sociolinguistique. Le cahier des charges que la section s'est donné indique pourtant clairement, à propos de la DDF (FLE et FLM), que « ces domaines de recherche font partie du champ de la $7^{\mathrm{e}}$ section, à condition que le cadre théorique convoque au moins un autre domaine des sciences du langage. On peut citer, à titre d'exemples, les recherches sur l'analyse des interactions pédagogiques, les recherches sur l'acquisition, les recherches sur la didactique de l'oral fondées sur la phonologie ou l'analyse conversationnelle, les recherches sur l'illettrisme ou sur le français langue seconde appuyées sur la sociolinguistique, les recherches analysant les discours officiels avec les outils de l'analyse de discours, les recherches sur l'intégration des TICE convoquant l'analyse de discours et la sémiologie, etc. Certaines thèses examinées non seulement ne font pas mention d'un tel cadre théorique mais encore ne tiennent aucun compte des recherches ancrées en sciences du langage existant dans le domaine qu'elles abordent : la qualification ne peut alors qu'être refusée. » (Compte rendu... 2005).

\section{Conclusion}

Dans leur présentation de Pratiques 145-146, A. Halté et A. Petitjean, se fondant surtout sur la réforme de la formation des maîtres et sur l'intégration des IUFM dans les universités, font le constat suivant : «le contexte politique et institutionnel qui est le nôtre en France n'est pas spécialement favorable aux recherches en didactique » $(2010: 3)$. Le rapide aperçu que nous avons donné des pratiques de la $7^{\mathrm{e}}$ section du CNU en matière de qualification des candidats spécialistes de didactique du français et des langues - c'est un autre aspect important du 
contexte institutionnel -, nous paraît de nature à nuancer ce constat. Car, s'il est exact que les candidatures relevant de ce domaine font plus souvent l'objet d'un refus de qualification que dans la majorité des autres domaines, cela tient d'abord - nous semble-t-il - au recours à des critères d'appartenance au champ et assez peu à une stigmatisation des recherches en didactique ou à une méconnaissance des normes de scientificité qui y prévalent, ne serait-ce que parce que les didacticiens sont bien représentés au sein de la section. La question de savoir s'il faut changer ces critères ou bien si les directeurs de thèse et les doctorants du domaine doivent mieux les prendre en compte quand ils visent une qualification par la $7^{\mathrm{e}}$ section, demeure ouverte.

\section{Références bibliographiques}

ARRIVÉ, M. (2000) : « Être linguiste aujourd'hui, qu'est-ce à dire ? Notes démographiques et autres sur la profession de linguiste », Buscila 52, 2/2 : 2-8.

Berré, M., SAVATOVSKY, D. (éds). (2010) : « De l'École de préparation des professeurs de français à l'étranger à l'UFR DFLE (1920-2008)», Documents pour l'histoire du français langue étrangère et langue seconde, 44.

Chevalier, J.-C., ENCREVÉ, P. (2006) : Combats pour la linguistique de Martinet à Kristeva. Essai de dramaturgie épistémologique, Lyon, ENS Éditions.

COSTE, D. (2011) : «À propos d'un manuel français de linguistique appliquée », in J.-L. Chiss, D. Savatovsky (éds), Disciplinarisation des savoirs linguistiques. Histoire et épistémologie (Actes du Colloque international SHESLHTL 2010, Paris, 29-30 janvier 2010). À paraître in Dossiers d'HEL 5 [Supplément électronique à la revue Histoire Epistémologie Langage].

GODELIER, M. (dir.) (1982) : Les Sciences de l'homme et de la société en France. Analyse et propositions pour une politique nouvelle, Ministère de la Recherche et de l'Industrie, Paris, La Documentation Française.

- (2002) : L'État des sciences de l'homme et de la société en France et leur rôle dans la construction de l'espace européen de la recherche, http://www2.enseignementsup-recherche.gouv.fr/rapport/archives.htm

Halté, A., Petitjean, A. (2010) : « Présentation. État des recherches en didactique du français. 1. Les pratiques enseignantes », Pratiques 145-46 [« Didactique du français $1 »]: 3-7$.

SAVATOVSKY, D. (2007) : «Classement par domaines de spécialité des recherches doctorales en sciences du langage d'après le titre des thèses figurant dans les dossiers de candidature à une qualification maître de conférences $\left(\mathrm{CNU}-7^{\mathrm{e}}\right.$ section - session 2006 », Buscila-Infos (nouvelle série) $18: 2-5$. 